To appear in Biology and Philosophy

This version my slightly differ from the final publication

\title{
From survivors to replicators: Evolution by natural selection revisited
}

To appear in Biology and Philosophy

Pierrick Bourrat

The University of Sydney,

Faculty of Arts,

School of Philosophical and Historical enquiries

Department of Philosophy

Main Quadrangle A14

Sydney, NSW 2006, Australia

Email: p.bourrat@gmail.com

\section{Acknowledgements}

I am thankful to Samuel Baron, Michael Duncan, Paul Griffiths, Johann Hariman, Adam

Hochman, Robyn Kath, Arnon Levy, Kristie Miller, Mark Olson, Maureen O’Malley, Susanna

Saracco, Kim Sterelny, Simon Varey, Elena Walsh, Michael Weisberg and two anonymous

referees for their comments on an earlier version of this paper. I am especially grateful to Michael Duncan who proofread the English of the paper. I would also like to thank Peter Godfrey-Smith for discussions on the subject. This research was supported under the Australian Research Council's Discovery Projects funding scheme DP0878650 and an International Postgraduate Research Scholarship from the University of Sydney. 
To appear in Biology and Philosophy

This version my slightly differ from the final publication

\section{From survivors to replicators: Evolution by natural selection revisited}

\section{Abstract}

For evolution by natural selection to occur it is classically admitted that the three ingredients of variation, difference in fitness and heredity are necessary and sufficient. In this paper, I show using simple individual-based models, that evolution by natural selection can occur in populations of entities in which neither heredity nor reproduction are present. Furthermore, I demonstrate by complexifying these models that both reproduction and heredity are predictable Darwinian products (i.e. complex adaptations) of populations initially lacking these two properties but in which new variation is introduced via mutations. Later on, I show that replicators are not necessary for evolution by natural selection, but rather the ultimate product of such processes of adaptation. Finally, I assess the value of these models in three relevant domains for Darwinian evolution.

\section{Introduction}

Since Darwin, a number of “recipes", to use Godfrey-Smith's terminology (2009), have been proposed to delimit the phenomenon of evolution by natural selection (ENS). Each of these recipes has three essential ingredients, in so far as they each claim that, to exhibit ENS, a population of entities must have 1) variation, leading to 2) differences in fitness and 3) a form of heredity for this variation (for different recipes see Brandon 1990; Darwin 1859; Endler 1986; Lewontin 1970, 1978, 1985; Okasha 2006; Ridley 1996). These three ingredients are often conceived as both necessary and sufficient for ENS and applying not only to biological populations, but to virtually any population of entities. Although it is not emphasized by most authors (for an exception see Ridley 1996), a condition of reproduction is presupposed by each of these recipes. This is because the concept of heredity entails the concept of reproduction. In this paper I explore the links between reproduction, heredity and ENS. One aim of the paper is 
To appear in Biology and Philosophy

This version my slightly differ from the final publication

to demonstrate that, strictly speaking, ENS can occur in populations of entities that do not reproduce and as a consequence heredity is not required for ENS. This point has already been argued forcefully by Bouchard in different places $(2004,2008,2011)$ but only verbally. I will argue that ENS in non-reproducing and reproducing populations is one and the same process based on the use of models. But, as such, this claim seems to undermine the crucial role of reproduction and heritability in Darwinian processes. Thus a second, more important, aim of the paper will be to show that reproduction and heredity, despite not being essential components of ENS, do have a special role in Darwinian processes; namely that they can be primordial adaptations of non-reproductive Darwinian populations, indispensable for complex cumulative adaptations (such as the human eye or brain). Finally, I will outline a new interpretation of the concept of fitness in light of these arguments that will partially vindicate Bouchard's approach on fitness.

The rest of the paper is divided into four sections. In Section 2 I show that ENS or a process close enough to ENS (weak ENS), can occur in populations of entities that do not reproduce. I thus argue that heredity and reproduction are not essential components of ENS. Although this represents a serious problem for a foundational description of the process of ENS, I concur with Okasha (2006) that this form of ENS is evolutionarily uninteresting since populations of nonreproducing entities cannot lead to adaptations as classically understood by evolutionary biologists. At that point it will become clear that the different recipes of ENS describe one particular form of ENS that allows for cumulative evolution and complex adaptations. I will call this particular form of ENS: paradigmatic ENS. From then on, I ask whether paradigmatic ENS can originate from weak ENS or whether the two forms of ENS are due to two fundamentally different processes of natural selection.

In Sections 3 and 4, I answer this question by showing, using individual-based models that paradigmatic ENS not only can but is likely to originate from weak ENS. These two sections 
To appear in Biology and Philosophy

This version my slightly differ from the final publication

present a diachronic account of ENS in which the third ingredient, heredity (including reproduction), is not taken for granted, but rather "endogeneized" into a broader account of ENS. Endogeneizing paradigmatic ENS will necessitate four cumulative steps. a) New variation must be introduced in the population over time. Without it, no cumulative evolution at all is possible. This will be postulated in all my models. b) At least some members of this population must be able to reproduce for otherwise the population goes extinct. I show how that is possible in Section 3. c) Given that the population is able to maintain its size, all the entities of the population must be able to reproduce and d) differences in fitness must be transmitted from parents to offspring. I show how these two phenomena are possible in Section 4. At the end of Section 4 it will become clear that heredity is actually a complex adaptation of Darwinian populations in which originally only weak ENS is possible. Most of my reasoning will be based on simulations of individual-based models using the software NETLOGO 5.02 (Wilensky 1999). Using simulations will have two virtues. First, it will help the reader to see the different concepts and processes I explore "in action". Second, it will allow me to develop models that although simple, are too complex to be expressed verbally efficiently.

In Section 5, I assess the value of the endogeneization-of-heredity hypothesis in three domains of relevance for the concept of ENS : origins of life, major transition and cultural evolution.

\section{ENS without reproduction}

\footnotetext{
${ }^{1}$ I borrow this term and idea from Okasha (2006) and his discussion on the levels of selection in which he explains that multicellular organisms once taken for granted by individual level selection theory have been "endogeneized" by the multilevel selection theory, which makes of multicellularity a product of natural selection.
} 
To appear in Biology and Philosophy

This version my slightly differ from the final publication

Existing recipes postulate heredity as a necessary condition for ENS. Yet, one can easily find cases in which ENS or a very similar process is observed, but in which there is no reproduction. Take this example given by Van Valen $(1989,2)$ :

"[Granite] is composed mostly of grains of feldspars and quartz, with some mica and other minerals inserted among them. When granite weathers, the feldspars and micas become clays but nothing much happens to the quartz grains. They are most resistant and get transported down streams or along shores. Thus most beaches are the result of differentially eroded granite. This is an example of natural selection in the nonliving world. Quartz grains survive longer than feldspar grains, and there is a progressive increase in the average resistance to weathering, of the set of grains that have still survived $[\ldots]$ The lack of reproduction imposes constraints on the flexibility of evolution here, but one shouldn't confuse that with the selection itself."

Although grains of quartz, feldspars and mica do not reproduce in any reasonable sense of the word, there is a form of selection on grains of minerals which leads to an overall change in the population. This type of selection is called viability selection or more generally selection on persistence. I call entities that only undergo this type of selection "survivors". The fact that a form of ENS can be observed in populations of survivors represents a serious problem for standard recipes of ENS. These recipes supposedly capture the essence of ENS; yet, it seems as if they do not recognize viability selection in populations of survivors as resulting in ENS. Why is this case?

One direct response to this question, given by Godfrey-Smith $(2009,40)$, is that, although an extension of the Darwinian apparatus to populations of survivors is possible, it is very artificial. By "artificial", I take it Godfrey-Smith means that historically, the concept of natural selection has only been applied to (living) entities that do reproduce. Applying these ideas to entities that do not reproduce is, in this sense, artificial because the theory was not elaborated with nonreproductive entities in mind. Although it might be historically artificial to extend the concept of ENS to populations of entities that do not reproduce, artificiality cannot be a strong argument 
To appear in Biology and Philosophy

This version my slightly differ from the final publication

against a foundational description of Darwinian processes or what Griesemer calls generalization by abstraction of Darwinian evolutionary theory (Griesemer 2005). If recipes of ENS represent such foundational descriptions, they should be able to account for the simplest cases.

Another answer comes from Okasha $(2006,214)$ in his discussion of clade selection. He uses the term weak selection when referring to processes of selection between different types of survivors (because they do not "reproduce," clades are a type of survivor in my terminology). Okasha claims that the concept of natural selection is interesting and can lead to adaptations only when applied to entities that reproduce. If selection in populations of survivors cannot lead to adaptations, it might be justified to separate cases of differential persistence (weak ENS,) from cases of paradigmatic ENS.

Some people even refuse to grant differential persistence or viability of types the status of evolution, let alone ENS. Whether we should call the phenomenon resulting from weak selection "evolution" is in fact disputable. I am inclined to grant it the status of ENS because changes in frequency of types or variants due to the sole action of selection are usually identified by population geneticists as evolution. But essentially this problem is mostly a terminological one and is not what I am interested in here. The only important question I want to answer is whether weak ENS (or whatever one wants to call it) and paradigmatic ENS are two fundamentally distinct processes. As we saw, Okasha considers weak ENS uninteresting because it cannot lead to adaptations. This seems to be the kind of fundamental difference between the two forms of ENS we are looking for. But is this proposition correct? Is it correct to claim that there are no adaptations under a process of weak ENS? This mostly depends on what Okasha means by adaptation. Brandon $(1990,40)$ proposes distinguishing abistorical adaptations, ${ }^{2}$ which simply refer to properties that increase the fitness of their possessors, from "adaptation as products of the

\footnotetext{
${ }^{2}$ A synonym for ahistorical adaptation is "adaptiveness"
} 
To appear in Biology and Philosophy

This version my slightly differ from the final publication

process of ENS" (referred to later as genuine adaptations). I take it that what Brandon means by a "product of the process of ENS" is something similar to what Godfrey-Smith calls the "positive role of natural selection" (Godfrey-Smith 2009, 49-50). In his discussion on origin and distribution explanations, Godfrey-Smith proposes that in spite of its "negative" role (i.e. eliminating variation over time) natural selection has a positive, creative role in evolution. It does so by "changing the population-level background against which new mutations can occur" (Godfrey-Smith 2009, 50).

The following is a schematic way to represent the process behind changes in population-level background against which new mutations can occur. In a population of entities that reproduce in which there is variation with regard to one trait, selection (let us assume it is directional) will eliminate most of the variants, keeping only the fittest ones. These variants will then reproduce and transmit their phenotype to their offspring, so that the successful phenotypes become relatively more highly represented in the overall population. New advantageous mutations further increasing the fitness of their bearers will thus become more probable. Godfrey-Smith argues that this is the kind of phenomenon that leads to complex adaptations such as the human eye or brain. That is, I take it, the kind of adaptation Okasha claims is not possible in populations of survivors. Ahistorical adaptations are, however, perfectly possible in these populations.

In some respects Okasha is right. Genuine adaptations cannot appear in population of survivors, but not because of some fundamental distinction between a population of survivors and a population of reproducing entities. The reason why they cannot appear is because the probability of successive "lucky" mutations is so low in those populations that they become nearly impossible. Going back to Van Valen's quote, a low probability of successive advantageous mutation is the constraint on the flexibility of evolution the lack of reproduction imposes on populations. Yet, in principle with luck or with an enormous population size 
To appear in Biology and Philosophy

This version my slightly differ from the final publication

(assuming that some mutations occur), genuine complex adaptations could appear in a population of survivors. Thus, the concept of adaptation cannot separate the two forms of ENS. Is there any other way to separate them?

In the next two sections I argue that weak and paradigmatic ENS should in fact not be separated because paradigmatic ENS is a special case of ENS that not only can, but is likely to originate in populations of survivors with the ability to mutate. The reason for this is that heredity and reproduction can be themselves genuine complex adaptations in those populations. In fact moving from a population of survivors to a population exhibiting paradigmatic ENS will require four cumulative steps or conditions. The first step is a) that new variation is introduced in the population. Without it no cumulative evolution is possible. The second step is that $b$ ) the population is able to maintain its size or if the size of the population is not limited, to grow. This is not the cases in weak ENS, in which populations are ultimately condemned to extinction. But a population able to maintain its size does not make it a population in which paradigmatic ENS necessarily occurs. Two other important phenomena occur in paradigmatic cases of ENS: $c$ ) advantageous phenotypes are able to be transmitted during reproduction making populationlevel changes against which new mutations can occur possible, and finally $d$ ) reproduction is pervasive therefore each entity in the population is in principle able to reproduce. Invasion of one variant in the population of reproducing entities under the sole action of natural selection is the hallmark of a process of genuine adaptation.

In the next section I build three individual-based models that will show how one can move from a population of survivors to a population of entities able to maintain its size and thus meet conditions $a$ and $b$. I will distinguish the concept of procreation, which is reproduction without heredity, from the concept of minimal reproduction, which is indefinite procreation i.e. procreation being transmitted from parents to offspring. I will then show that minimal reproduction, but not procreation, once introduced in a population of survivors through mutation (a), leads this 
To appear in Biology and Philosophy

This version my slightly differ from the final publication

population to maintain its size $(b)$. Yet these conditions will be insufficient for paradigmatic ENS to occur. At that point I will propose an important distinction between heredity of and heredity on. In Section 4, using other models, I show how conditions $c$ and $d$ can be obtained from a population of entities able to maintain its size by introducing heredity on the ability to procreate (i.e. reproduction) and heredity on other Darwinian traits and why heredity on represents a genuine adaptation of those populations. Towards the end of Section 4, I propose that the existence of replicators (Dawkins 1982; Hull 1980) can result from a similar process of adaptation and thus can be conceived as a product of natural selection rather than condition for it.

\section{Reproduction and heredity in ENS}

How can we get a population which maintains its size from a population of survivors? It seems that the ability to reproduce is the right property to give to our survivors. But reproduction is a complex phenomenon that can be decomposed into two more fundamental phenomena: the first, which I name procreation, ${ }^{3}$ is reproduction without any property transmitted from parents to offspring including the ability to procreate; the second is inheritance from parent to offspring of the ability to procreate. A procreated entity of any given reference class inherits nothing from its parents but the facts of coming into existence and membership of that class. It might be difficult to imagine an act of procreation which would not involve the transmission of some properties other than the fact of coming into existence and the choice of a reference class is a well-known problem in statistics. Rotten meat could be said to 'procreate' maggots if one had in mind a theory of spontaneous generation with the class of reference "entities made of flesh". Although spontaneous generation theories are absent from modern biology, it should be clear

\footnotetext{
${ }^{3}$ I distinguish procreation from "progeneration" used by Griesemer (2000) which is in my terminology, procreation with material overlap.
} 
To appear in Biology and Philosophy

This version my slightly differ from the final publication

that procreation in a given class of objects does not necessarily involve inheritance or transmission of traits and that it is therefore the right concept we need for our purpose since we want to dissociate heredity from reproduction.

With the distinction between procreation and reproduction in mind, we can now start to imagine the fate of a population of survivors in which there is variation in viability and some of these survivors spontaneously mutate into procreators: entities able to procreate. Does this mutation allow our initial population of survivors to maintain its size?

To answer this question, I built a set of individual-based models. ${ }^{4}$ In their simplest form, individual-based models simulate the behavior of individuals that are "asked" every unit of time to behave in a certain way (e.g. to move, to reproduce, to die etc.). My models describe a population initially composed of survivors. Each survivor has only one property, its survival rate over each unit of time, which remains constant during its whole life and is fixed at birth. The survival rate of each survivor is chosen randomly between 0 and 0.99 (to avoid immortal survivors). Time is discrete. For every unit of time the population undergoes one selection event.

During the event of selection, each individual is selected according to its survival rate, also called viability. Selection operates as follows: if a given individual has a survival rate of 0.6 (recall that this value is chosen randomly at birth), this means that its chances of surviving this round of selection will be $60 \%$. The lower the survival rate, the lower the chances of the individual surviving until the next unit of time.

\footnotetext{
${ }^{4}$ The software NETLOGO 5.02 has been used. Two advantages of individual-based models (a microscopic approach to modeling) over macroscopic approaches are their flexibility (which makes them appealing for testing new hypotheses) and that they allow for each individual in the simulation to be unique. These two features make individual-based modeling ideal for the purpose of this article.
} 


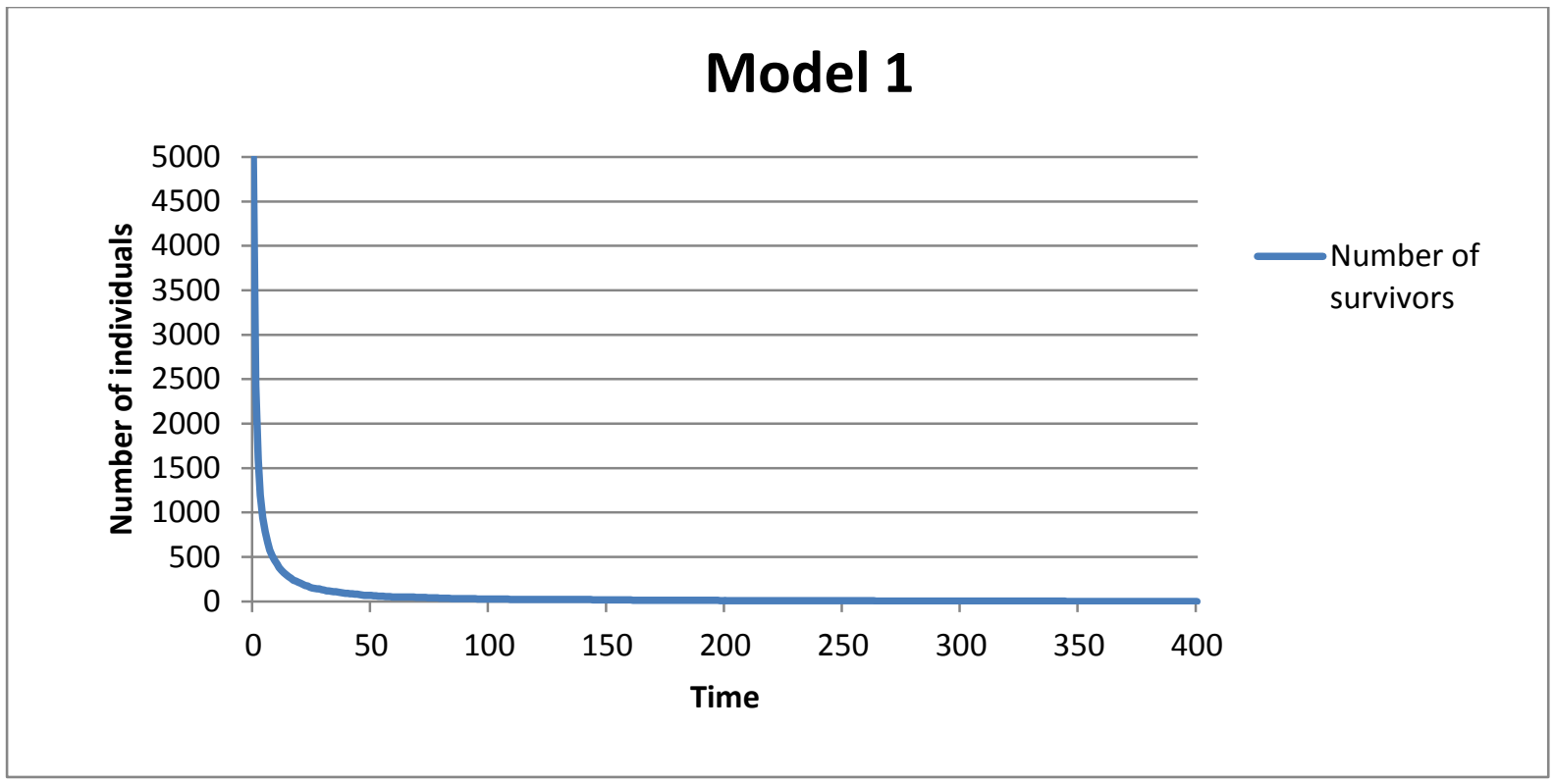

Figure 1. Simulation of Model 1 over 400 units of time

Model 1 is essentially similar to ENS without reproduction as proposed in Section 2. There are only survivors in the population and no mutants. Figure 1 represents the graph of one typical simulation of Model 1 in which the number of survivors is recorded over time. At $t=0,5000$ survivors are created. As expected the population rapidly goes extinct. Model 1 represents a null model in my framework upon which the following models are built. Table 1 summarizes the different models and associated concepts used in Section 2 and 3.

What happens now to this population if procreation is introduced by mutation of one survivor into a procreator? A second model (Model 2) was created to answer this question. Model 2 is very similar to Model 1 but procreation is introduced in the population through one mutant individual at the beginning of the simulation (see Table 1). When a survivor mutates into a procreator it acquires the possibility to procreate. ${ }^{5}$ Condition $a$ (that new variation is introduced into the population) is thus built into the model. Hence, procreators have two properties: a

${ }^{5}$ In Model 2 we assume that the mutation has already occurred. Mutation is thus exogenous to the model. 
To appear in Biology and Philosophy

This version my slightly differ from the final publication

survival rate within each unit of time (as do survivors) and a fertility rate (i.e. number of individuals procreated) within each unit of time as long as the procreator is alive. The fertility rate of a procreator remains constant throughout the whole life of the minimal reproducer unless further mutation occurs.

As in Model 1, every individual undergoes selection. But after selection procreators are able to procreate. ${ }^{6}$ During this stage each procreator, if any exist, procreates according to their fertility rate. Because there is no heredity on viability, the offspring's viability is chosen randomly between the minimal and maximal values allowed by the model (0 and 0.99). Because there is no heredity of the ability to procreate, offspring of procreators are survivors. After the stage of procreation, another stage is introduced in the model. I call it the check-for-overcrowding stage. During this stage, the size of the population is assessed and if it is higher than its initial size, individuals are randomly chosen and killed until the population roughly reaches its initial size. The sequence of events undergone by every individual in Model 2 is selection $\rightarrow$ reproduction $\rightarrow$ check-for-overcrowding; this restarts every unit of time.

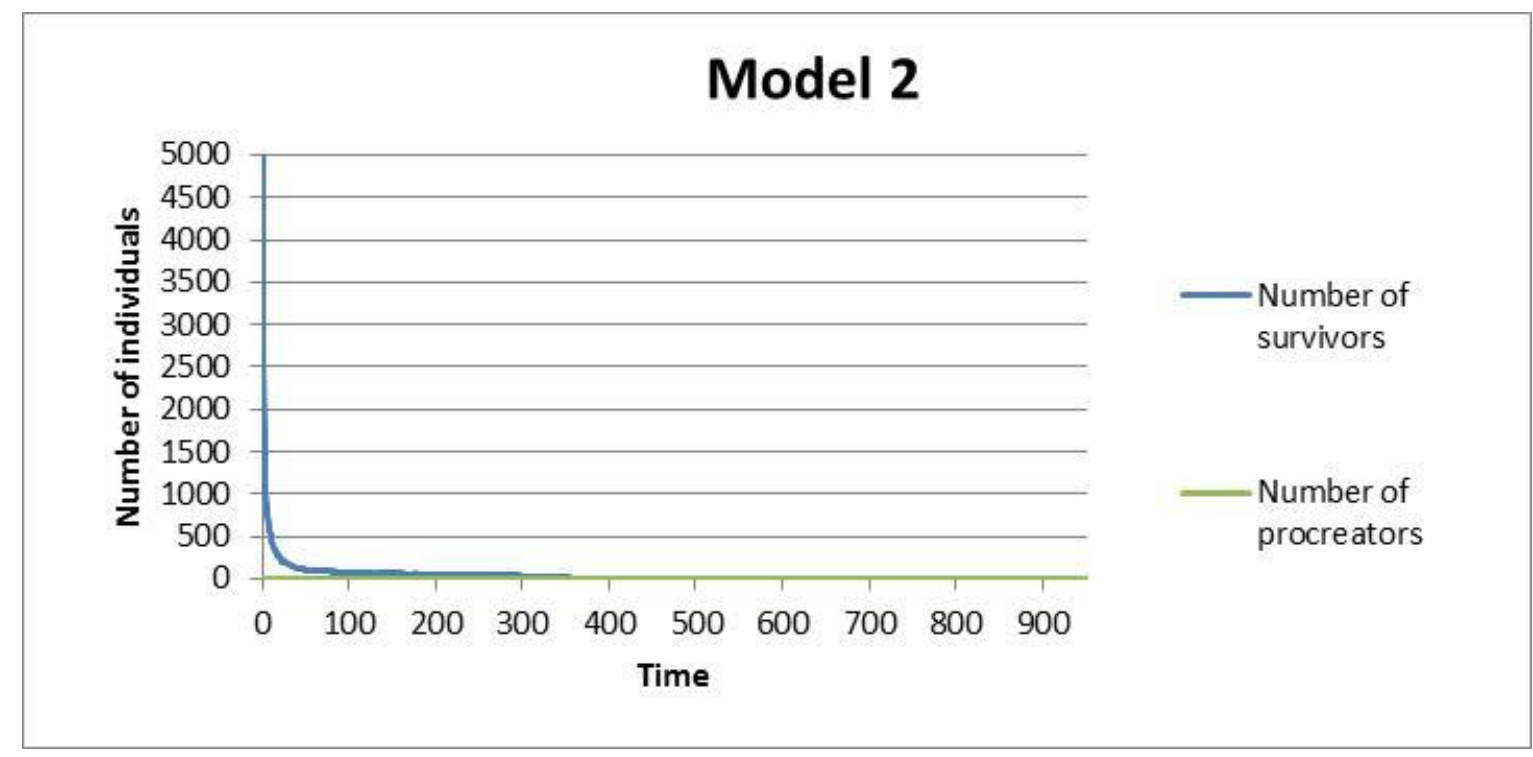

${ }^{6}$ In Model 2 and subsequent, procreation is always asexual. 
To appear in Biology and Philosophy

This version my slightly differ from the final publication

Figure 2a. Simulation of Model 2 over 900 units of time

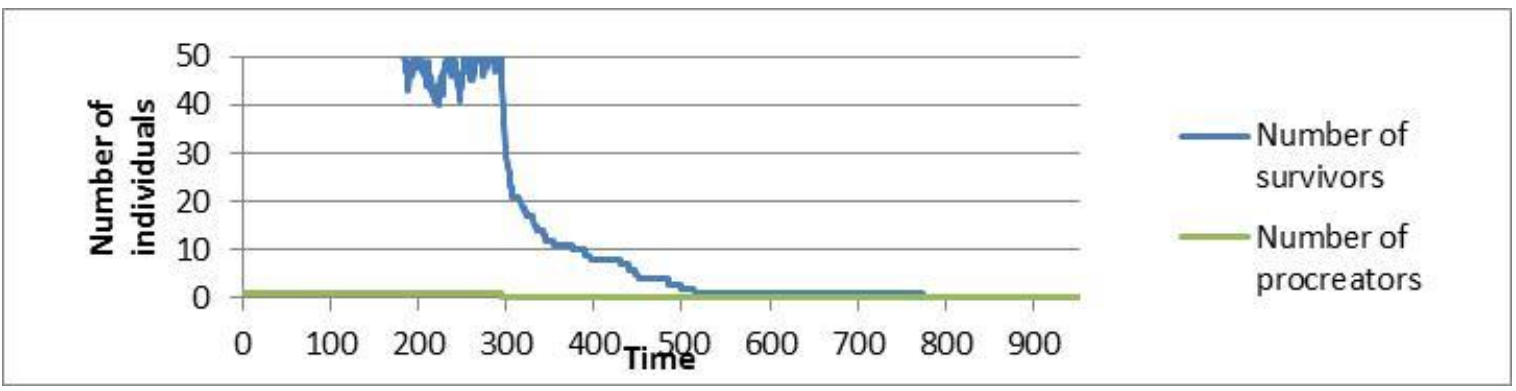

Figure 2b: Simulation of Model 2 over 900 units of time (zoom in)

Figure $2 \mathrm{a}$ and $2 \mathrm{~b}$ both represent the graph of one (and the same) typical simulation of Model 2 in which the number of survivors and procreators is recorded over time. At $t=0,4999$ survivors and 1 procreator which has mutated from a survivor are created. For the purpose of my argument, the viability and fertility of the mutant procreators are the highest possible allowed by the model(respectively 0.99 and 10) in order to maximize its fitness and see whether any change at all is possible when compared to a population of survivors only. As can be seen in figure $2 \mathrm{~b}$, the population becomes extinct after 700 units of time while the procreator before the $300^{\text {th }}$ unit of time. This is not a surprising result. Because procreators' offspring are survivors, the population is only able to produce new entities as long as the initial procreator is alive. Once this initial procreator dies, the population becomes similar to the population of survivors in Model 1. No matter how many procreators there are in the initial population, this population is ultimately condemned to extinction.

Thus, a population in which procreation has emerged is unable to maintain its size and the type of ENS observed in this population is very similar to weak ENS. I call it quasi-weak ENS. This result is good evidence that heredity must be part of the reason why a population is able to display paradigmatic ENS and more proximally to satisfy $b$, simply because without heredity of the ability to procreate, a population will become extinct. Let's now see what happens in our population if instead of a procreator the mutant introduced is a minimal reproducer. I define a 
To appear in Biology and Philosophy

This version my slightly differ from the final publication

minimal reproducer as a procreator which is able to transmit its ability to procreate to some, if not all, of its offspring. I call this entity a minimal reproducer because its ability to procreate is not transmitted to every one of its offspring.

In Model 3, I start as in Model 2 with a population of 4999 survivors and one mutant (in this case a minimal reproducer). Minimal reproducers behave in a similar way to procreators except that some of their offspring are also minimal reproducers. Thus, the only difference between Model 2 and Model 3 is that in Model 3 rules of transmission of fertility and of the ability to procreate between parent and offspring must be chosen (see Table 1). The rule of transmission of viability remains the same. The sequence of events undergone by every individual each unit of time in this model is the same as in Model 2.

I now introduce the distinction between heredity of and heredity on. When there is heredity of a trait, the only thing a parent transmits to their offspring is the trait itself. The offspring's phenotype associated with this trait is uncorrelated to the phenotype of the parent and thus can take any possible value on that trait. When there is heredity on (which presupposes heredity of) a given trait, there is a correlation between the parent's and the offspring's phenotype on that trait. In a case of heredity of no prediction of the offspring's phenotype from the parent's phenotype is possible while some level of prediction is possible in a case of heredity on ${ }^{7}$ To make this clear imagine that an entity of size $S$ produces 10 offspring and that the scope of possibility on size of a given entity of the class of reference is between 1 and 10. If there is only heredity of size, the size of the offspring will be uncorrelated with the size $S$ of the parent. If there heredity on size, the offspring will be more likely to have size $S$ than any other size between 1 and 10 .

${ }^{7}$ Another way to make the distinction is by using the well-known distinction between determinable and determinate (Sanford 2011). Heredity of is a determinable property while heredity on is a determinate property 
To appear in Biology and Philosophy

This version my slightly differ from the final publication

Because there is no heredity in my models, except in relation to the ability to procreate in Model 3, the rule of transmission for fertility is that each minimal reproducer produced receives a fertility rate chosen randomly between the minimal (0) and maximal (10) fertility rate set by the model. The rule of transmission of the ability to procreate can be more difficult to choose. In fact, by introducing minimal reproducers in the model, there can be heredity of the ability to procreate, with or without heredity on this ability. When there is heredity of the ability to procreate, the only thing we can predict from a minimal reproducer, knowing its rate of transmission, is the proportions of its offspring that will be minimal reproducers. To which degree they will receive this ability - i.e. the proportion of their own offspring that will receive the ability - is unpredictable. I call minimal reproducers with this ability: unreliable minimal reproducers. On the other hand, when there is heredity on the ability to procreate, not only can we predict the proportion of offspring that will receive this ability but we can also predict, with a probability higher than chance, what level of reproductive ability each of the offspring will receive. This probability will itself depend on how reliable the transmission between parent and offspring is. I call minimal reproducers with this ability: reliable minimal reproducers.

In Model 3 heredity of the ability to procreate is introduced without heredity on the ability to procreate in order to see whether this simpler form of heredity is sufficient to make it such that our population is able to maintain its size. I set heredity of the ability to procreate at a maximal level of 0.20 so that a minimal reproducer cannot have more than $20 \%$ of its offspring as minimal reproducers. The level at which a minimal reproducer's offspring receives this ability is chosen randomly between 0 and 0.2 . I have set the maximal level of heredity very low in order see whether even low heredity of the ability to procreate is sufficient for a population to maintain its size and thus satisfy $b$. 


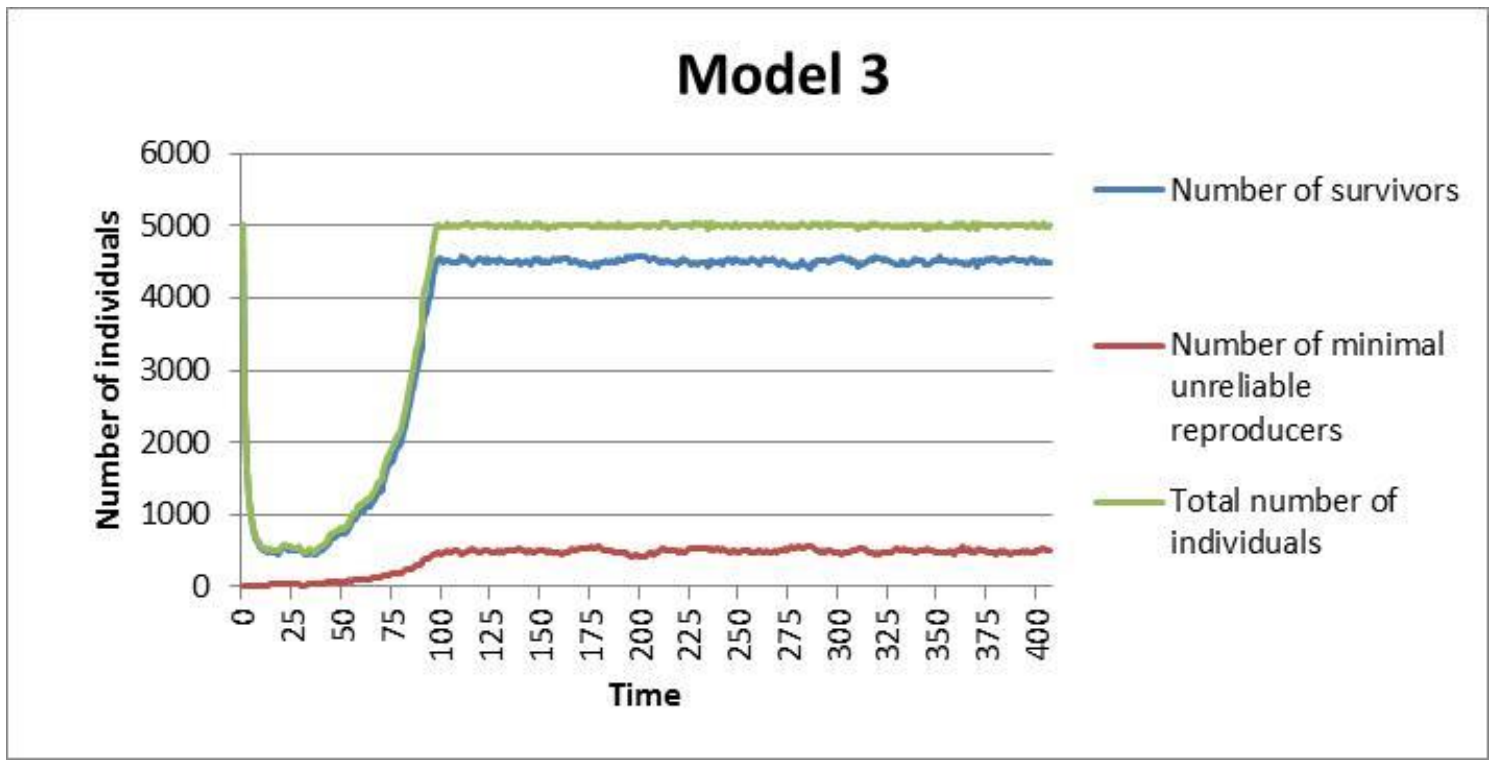

Figure 3a. Simulation of Model 3 over 400 units of time

Figure $3 \mathrm{a}$ represents the graph of a typical simulation of Model 3 in which the number of survivors, minimal reproducers, as well as the total number of individuals is recorded over time. At $\mathrm{t}=0,4999$ survivors and 1 minimal reproducer, which has mutated from a survivor, are created. As in Model 2, for the purpose of my argument in this simulation the survival rate, fertility rate and transmission rate of the ability to procreate of the mutant minimal reproducer are the maximum possible allowed by the model (respectively $0.99,10$ and 0.20 ) in order to maximize its fitness and maximize the chance of observing a difference between this model and the previous ones. The graph shows that during the first 15 units of time (in this particular simuation) the population behaves in similar way as in the two previous models: the population size drastically decreases. However, in contrast to the previous models, the population does not go extinct. After some time, the population size starts to increase until it reaches the maximal size around the $100^{\text {th }}$ unit of time. At that point the size of the population remains stable and is composed of around 10\% minimal reproducers.

We can conclude from Model 3 that heredity of the ability to procreate is sufficient for a population to maintain its size. It thus satisfies $b$, one of the three remaining requirements for a 
To appear in Biology and Philosophy

This version my slightly differ from the final publication

population to be able to exhibit paradigmatic ENS. But we have not yet established whether it satisfies $c$, i.e. the requirement that there be change in population level background against which new mutations can occur and $d$, i.e. the requirement that every individual of the population is able to reproduce, is not met.

One way to see whether $c$ is satisfied is to check whether individuals with the highest fitness are able to invade the population. If they do, this will represent a change in the population-level background against which new mutations can occur. But measuring fitness in our population is problematic. We have two types of entities, survivors and unreliable minimal reproducers, with different behaviors. Fitness is usually measured as the product of viability and fertility (Sober 2002). This definition of fitness cannot be applied to survivors because their fertility is nil (see [blind reference] for more on this problem). Yet, as we have seen earlier, some survivors are fitter than others since they persist longer. The fittest survivors are those with a survival rate of 0.99. The fittest minimal reproducers are also those with the highest viability. Importantly, there is no fertility selection in the model. Each minimal reproducer has on average 5 (between 0 and 10) offspring per unit of time independently of its phenotype if it survives until reproduction.

Viability selection is thus the only form of selection in the population regardless of whether the population contains survivors or minimal reproducers. This means that in Model 3, viability is the only parameter influencing the fitness of an entity. Therefore to evaluate whether entities with the highest fitness are able invade the population we need to see whether the variants of each type (i.e. survivor and minimal reproducer) with the highest viability allowed by the model (in this case 0.99 ) are able to invade the population. 
To appear in Biology and Philosophy

This version my slightly differ from the final publication

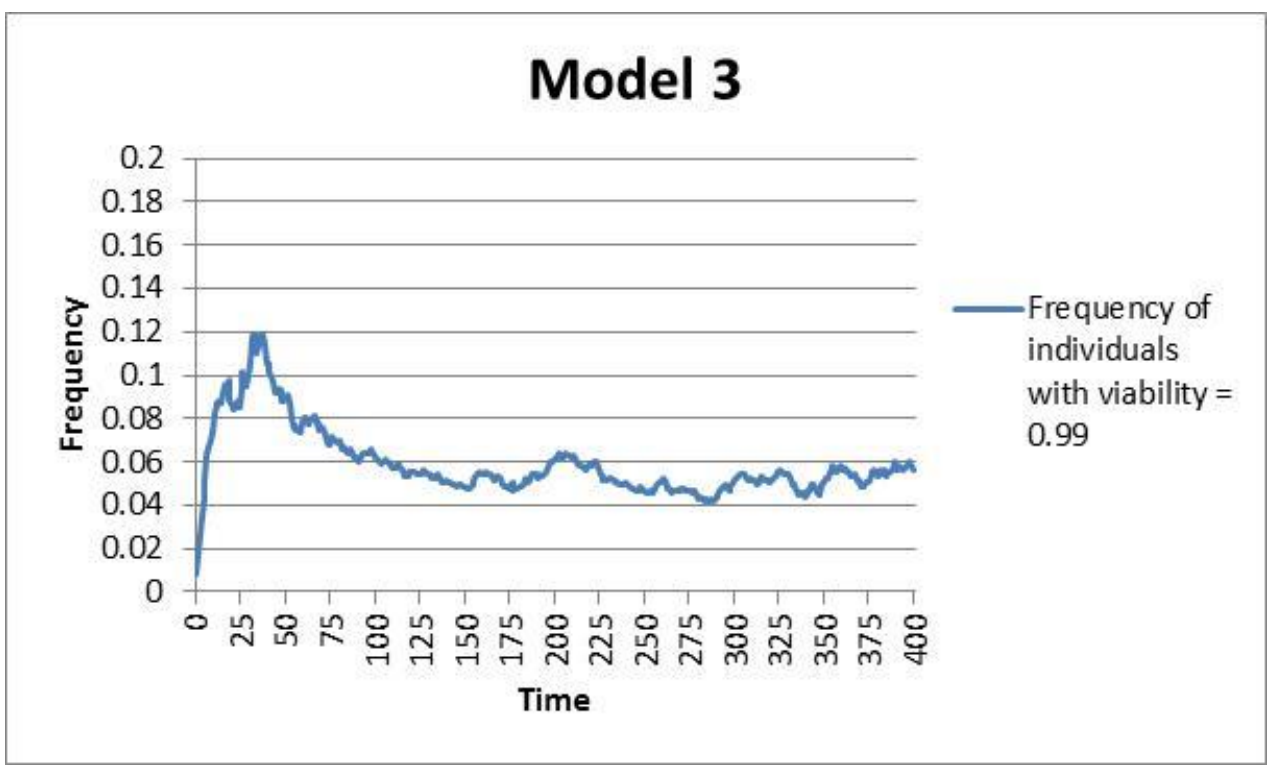

Figure 3b. Simulation of Model 3 over 400 units of time

Figure 3b represents a typical simulation of Model 3 over 400 units of time in which the proportions of entities with a viability of 0.99 is measured. The figure shows that the proportion of entities with a survival rate equal to 0.99 , very low at $\mathrm{t}=0$, increases and reaches an equilibrium around 0.05 around $t=130$. Thus in this model the population is not invaded by the fittest variants of each type. This is explained by the fact that there is no heredity on any properties in this set up, and successes and failures in terms of survival are not recorded by natural selection. Each survivor procreated by a minimal reproducer has the same chance of having a low viability as a high one, in spite of the fact that most of the survivors procreated come from minimal reproducers with high viability. Essentially the form of ENS observed in Model 3 is not very different from weak ENS apart from the fact that there is minimal reproduction in the model. I name it minimally-reproductive ENS and it does not satisfy $c$.

\begin{tabular}{lllrl} 
M Property bore & Description of the new & Entities in Type of ENS observed \\
o by the entities & property introduced by the the & \\
d apart from & Model & & & \\
e viability & & & population & \\
1 & & NA & Survivors & Weak: With complete exhaustion \\
\hline 1 & None & NA & &
\end{tabular}


To appear in Biology and Pbilosophy

This version my slightly differ from the final publication

\begin{tabular}{|c|c|c|c|}
\hline & & & of the population \\
\hline 2 Procreation & $\begin{array}{l}\text { Reproduction without any } \\
\text { heredity, including heredity of } \\
\text { the ability to procreate }\end{array}$ & $\begin{array}{l}\text { Survivors } \\
\text { Procreator }\end{array}$ & $\begin{array}{l}\text { Quasi-Weak: With complete } \\
\text { exhaustion of the population but } \\
\text { over a longer period of time than } \\
\text { with survivors }\end{array}$ \\
\hline $\begin{array}{ll}3 & \text { Unreliable } \\
\text { minimal } \\
\text { reproduction }\end{array}$ & $\begin{array}{l}\text { Heredity of the ability to } \\
\text { procreate. The level of the } \\
\text { ability to procreate is inherited } \\
\text { at an unpredictable level. }\end{array}$ & $\begin{array}{l}\text { Survivors } \\
\text { Unreliable } \\
\text { minimal } \\
\text { reproducers }\end{array}$ & $\begin{array}{l}\text { Unreliable-minimally- } \\
\text { reproductive: size of the } \\
\text { population self-maintained, but } \\
\text { changes in the background against } \\
\text { which new mutations can occur } \\
\text { are impossible. }\end{array}$ \\
\hline $\begin{array}{l}\text { Reliable } \\
\text { minimal } \\
\text { reproduction } \\
\text { / } \\
\text { Reproduction }\end{array}$ & $\begin{array}{l}\text { Heredity of and on the ability } \\
\text { to procreate. The level of the } \\
\text { ability to procreate is inherited } \\
\text { at a predictable level. } \\
\text { Successive mutation increasing } \\
\text { the level of transmission } \\
\text { (heredity of) and fidelity } \\
\text { (heredity on) of the ability to } \\
\text { procreate can be selected. } \\
\text { Heredity of and on the ability } \\
\text { to procreate reach a maximum. }\end{array}$ & $\begin{array}{l}\text { Survivors } \\
\text { Reliable } \\
\text { minimal } \\
\text { reproducers } \\
\text { then } \\
\text { Reproducer } \\
\text { s only }\end{array}$ & $\begin{array}{l}\text { From reliable-minimally-reproductive } \\
\text { to reproductive: as in Model } 3 \text { but } \\
\text { with possible changes in the } \\
\text { background against which new } \\
\text { mutations can occur. } \\
\text { Reproduction is a cumulative } \\
\text { adaptation resulting from } \\
\text { successive mutation on the level } \\
\text { of transmission and fidelity of the } \\
\text { ability to procreate. }\end{array}$ \\
\hline 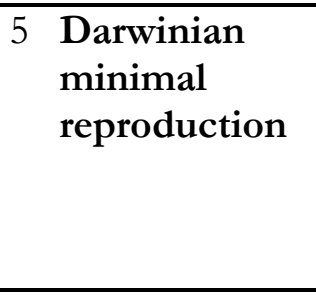 & $\begin{array}{l}\text { Heredity of the ability to } \\
\text { procreate. Heredity on one or } \\
\text { more traits having } \\
\text { consequences on the viability } \\
\text { or fertility on the entities } \\
\text { bearers of these traits }\end{array}$ & $\begin{array}{l}\text { Survivors } \\
\text { Darwinian } \\
\text { Reproducer } \\
\mathrm{s}\end{array}$ & $\begin{array}{l}\text { minimally-reproductive and cumulative: } \\
\text { complex cumulative adaptations } \\
\text { are possible }\end{array}$ \\
\hline $\begin{array}{l}6 \text { Reproduction } \\
\text { / } \\
\text { Replication }\end{array}$ & $\begin{array}{l}\text { Initially as in Model 5, } \\
\text { reproduction without heredity } \\
\text { on any other trait, but } \\
\text { apparition and selection of } \\
\text { successive mutation increasing } \\
\text { the heredity on traits with } \\
\text { consequences on the viability } \\
\text { or fertility of their bearers so } \\
\text { that Darwinian reproduction } \\
\text { appears. Heredity on these } \\
\text { traits reach the maximal level } \\
\text { of } 1 \text { so that Darwinian } \\
\text { reproduction becomes } \\
\text { replication }\end{array}$ & $\begin{array}{l}\text { Reproducer } \\
\text { s } \\
\text { then } \\
\text { Darwinian } \\
\text { reproducers } \\
\text { then } \\
\text { Replicators }\end{array}$ & $\begin{array}{l}\text { reproductive and cumulative i.e. } \\
\text { paradigmatic. complex cumulative } \\
\text { adaptations are possible }\end{array}$ \\
\hline
\end{tabular}

Table 1: The different concepts explored in Sections 2 and 3

\section{Heredity on and paradigmatic ENS}


To appear in Biology and Philosophy

This version my slightly differ from the final publication

Heredity of properties such as the ability to procreate or viability is thus insufficient for natural selection to record past successes and failures in reproducing populations and therefore to change the population-level background. In the following model (Model 4), taking the same setup as in Model 3, I introduce heredity on viability in order to evaluate whether it allows the entities with the highest viability to invade population and thus change the population-level background against which new mutations can occur (see Table 1). There is no straightforward way to choose which level of heredity on viability should be introduced. With perfect heredity on viability, the same survival rate is transmitted from the parent to the offspring. With a very low heredity, this viability is only slightly more likely to be transmitted than any other. For the sake of simplicity, I chose to introduce perfect heredity on viability in Model 4. The mutant initially present in the population is still an unreliable minimal reproducer because the ability to reproduce is not transmitted reliably from parent to offspring, but it is also what I call a Darwinian reproducer because its viability, one of the components of fitness (hence the qualification of Darwinian), is transmitted with a higher probability than chance to its offspring.

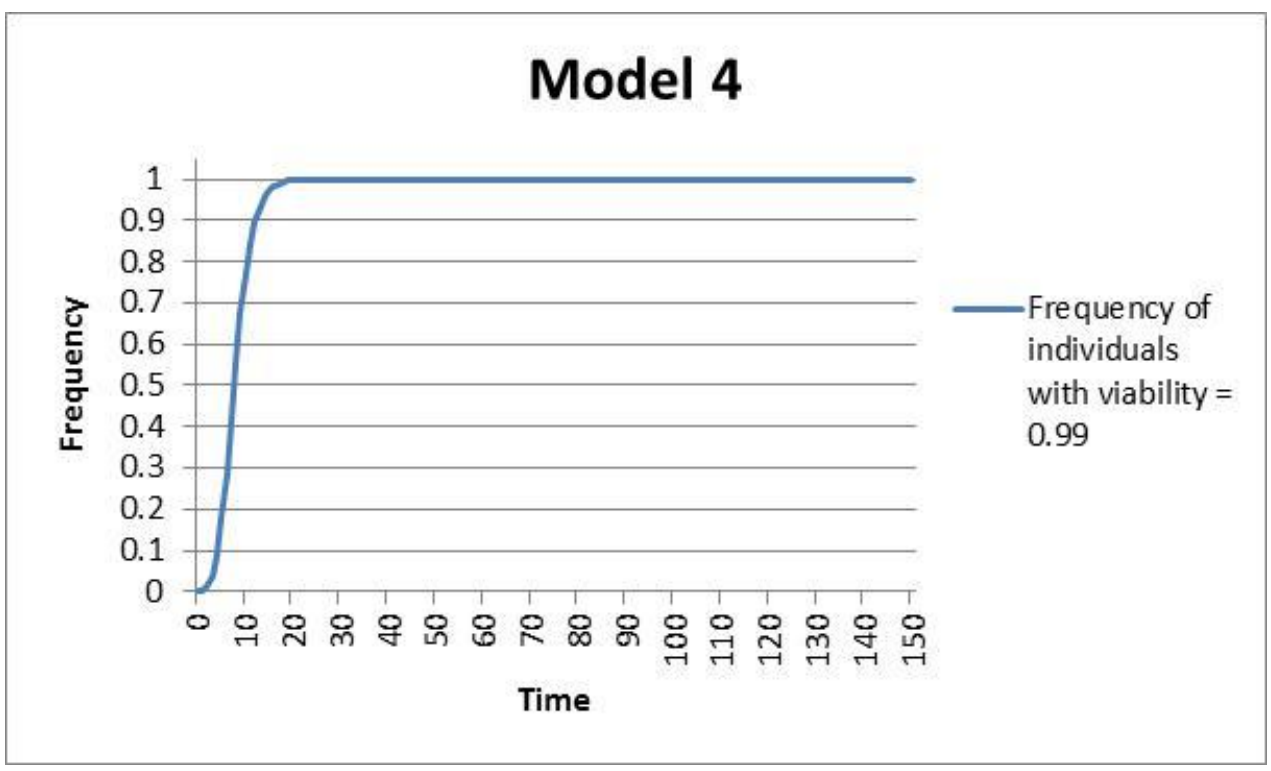

Figure 4. Simulation of Model 4 over 150 units of time 
To appear in Biology and Philosophy

This version my slightly differ from the final publication

Figure 4 represents the graph of a typical simulation of Model 4 over 150 units of time in which the proportion of entities with a viability of 0.99 is measured. The figure shows that the proportion of entities with a survival rate equal to 0.99 , very low at $\mathrm{t}=0$, increases and in contrast to Model 3 does not reach an equilibrium but rapidly invades the population. This is explained by the fact that, because there is heredity on viability in this set-up, successes and failures are now recorded by natural selection. Each survivor procreated by a minimal reproducer has the same viability as its parent. Because more procreated individuals (whether survivors or minimal reproducers) have a viability of 0.99 and the population is able to maintain its size, the variants with the highest fitness invade the population.

Thus, Model 4 shows that heredity on viability allows the population to satisfy $c$. In the population described by the model, individual changes brought about by advantageous mutations can be transmitted to the whole population. This is the mark of cumulative ENS and of populations able to display complex adaptations such as the human eye or brain. I call this form of ENS, minimally-reproductive cumulative ENS.. Yet, minimally-reproductive cumulative ENS is not reproductive cumulative ENS (i.e. paradigmatic ENS), in which there are no minimal reproducers anymore but reproducers only, all able to transmit indefinitely their ability to procreate to all their offspring. For a population to be able to exhibit paradigmatic ENS it must meet requirement $d$

With Model 5 I will show that high fidelity heredity on the ability to procreate is a cumulative or complex adaptation of mixed populations of survivors and minimal reproducers (i.e. initially exhibiting minimally-reproductive ENS) leading those populations to exhibit reproductive ENS (i.e. ENS in which every entity reproduces) and thus satisfy $d$. Although Model 4 showed that heredity on viability is an adaptation, it did not show how we can move from a population with no heredity on viability to a population with a high level of heredity on viability. That is what I 
To appear in Biology and Philosophy

This version my slightly differ from the final publication

will demonstrate with Model 6, in which both $c$ and $d$ are satisfied within one and the same population.

How can we get reproductive ENS from a mixed population of survivors and minimal reproducers? To answer this question, I start with the set-up used in Model 3 in which we have heredity of the ability to procreate (at a low level: 0.2 ) but no heredity on this ability. The result we observed was a population ultimately able to maintain its initial size but composed of a mix of survivors and minimal reproducers with very low levels of heredity of the ability to procreate. To have a population composed of reproducers only, two things must happen. First, the ability to procreate must be transmitted to every offspring of minimal reproducers - i.e. there needs to be heredity of the ability to procreate at the maximal level (1) resulting in the population becoming a population of reproducers. Second, each offspring procreated by a reproducer must receive the ability to procreate at the same level as its parent. In other words, there must be perfect heredity on the ability to procreate, the population thus becoming a population of reliable reproducers. Our question thus becomes: How can we move from a population similar to the population of Model 3 to a population with perfect heredity on the ability to procreate at a maximal rate, i.e. a population of reliable reproducers?

Model 5 shows how that is possible. There are two crucial differences between Model 3 and Model 5 (see Table 1). In Model 5, on top of the three stages (selection, reproduction and checking-for-overcrowding) undergone by each minimal reproducer, a fourth "mutation" stage is added before the selection stage so that the sequence of events undergone by every individual each unit of time is mutation $\rightarrow$ selection $\rightarrow$ reproduction $\rightarrow$ check-for-overcrowding. During the mutation stage, each minimal reproducer can mutate independently on two traits at a $10^{-3}$ rate. A mutation on the first trait increases or decreases the maximal rate at which an individual is able to transmit its ability to procreate (i.e. increases or decreases heredity of this ability). If the maximal rate of transmission of a minimal reproducer is 1 , it becomes a reproducer. A mutation on the second 
To appear in Biology and Philosophy

This version my slightly differ from the final publication

trait increases or decreases the fidelity at which the maximal ability to procreate of an individual is transmitted. If the fidelity of the transmission of the ability to procreate is nil, there is no heredity on the ability to procreate at a given level. If the fidelity is perfect, there is perfect heredity on the ability to procreate and unreliable reproducers become reliable reproducers.

As with Model 3, I initially set the maximal level of heredity of the ability to procreate at 0.2 for each minimal reproducer in the population. This means that at most, only $20 \%$ of the offspring of these minimal reproducers will also be minimal reproducers. The other $80 \%$ will be survivors. The fidelity at which each minimal reproducer can transmit its ability is initially nil, so that there is no heredity on the ability to procreate. This means that each procreated minimal reproducer will have initially a level of heredity of the ability to procreate randomly chosen between 0 and 0.2. At each event of mutation on the maximal level of the ability to procreate, a minimal reproducer chosen randomly in the population sees its maximal level of procreation increase or decrease by 0.01 with the same probability. Mutatis mutandis, at each event of mutation on the fidelity of transmission of the ability to procreate, a minimal reproducer chosen randomly sees the fidelity at which it will transmit its ability to procreate increase or decrease by 0.01 . All other parameters are kept identical to Model 3.

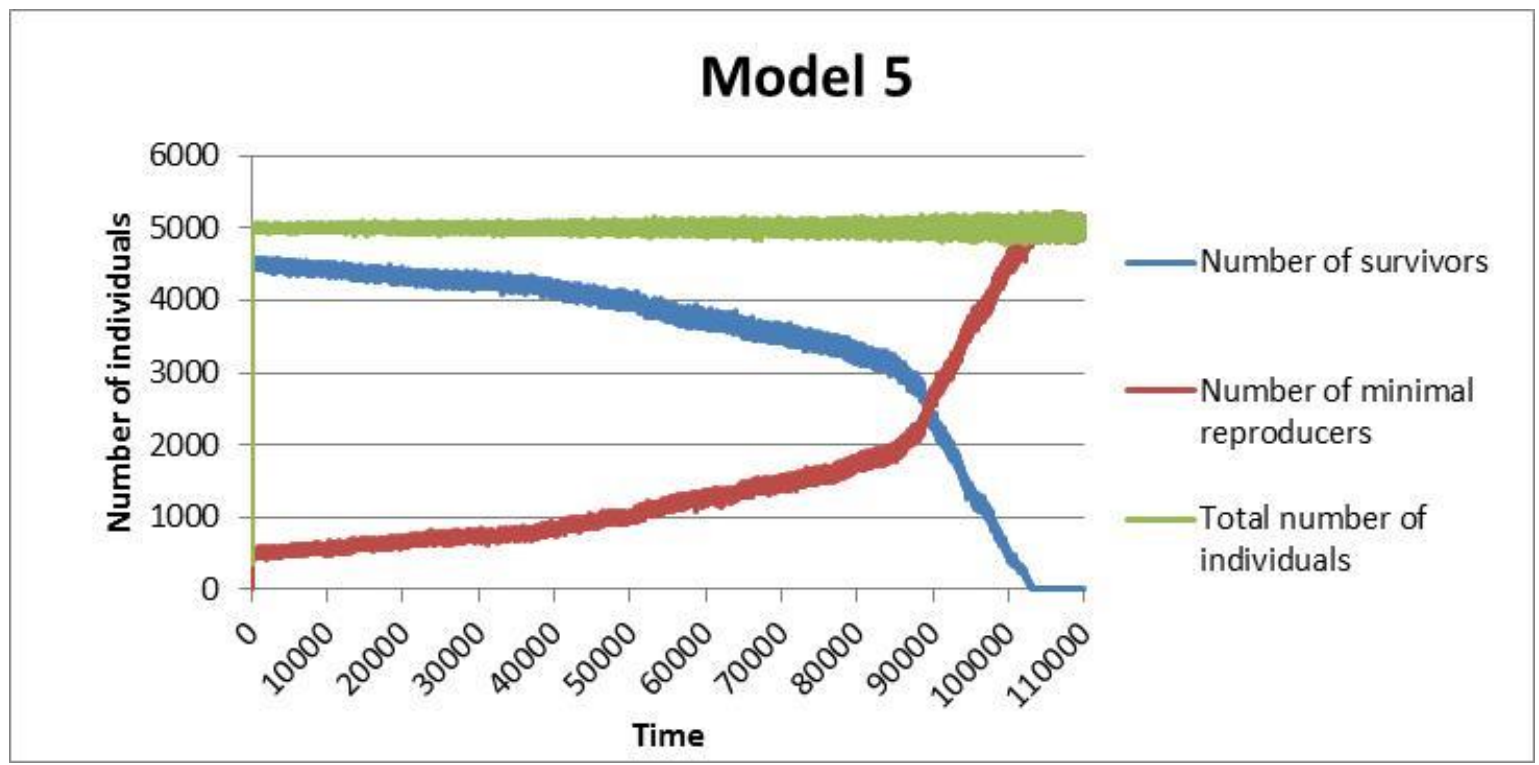


To appear in Biology and Philosophy

This version my slightly differ from the final publication

Figure 5 a. Simulation of Model 5 over 11000 units of time

Figure $5 \mathrm{a}$ represents a typical simulation of Model 5 in which the number of survivors and reproducers, as well as the total number of individuals is recorded over time. During the first units of time (which are not visible because figure $5 \mathrm{a}$ is not detailed enough) the total number of individuals rapidly decreases. The explanation is strictly the same as for the previous models: most survivors and minimal reproducers die because their survival rate is low. Only survivors and minimal reproducers with a high viability survive. Some of the minimal reproducers start procreating new minimal reproducers with a maximal rate of 0.2 , which in turn are able to reproduce. This explains why the population does not go extinct and starts increasing in size: more and more minimal reproducers are procreated. Figure 5b, shows that both the maximal level of heredity of the ability to procreate and the mean fidelity of heredity on the ability to procreate, starting respectively with a value of 0 and 0.2 , reach values superior to 0.99 after $\mathrm{t}=884000$ and $\mathrm{t}=104000$ respectively (in this simulation). This explains why at $\mathrm{t}=104000$, the population is composed of more than $99.5 \%$ minimal reproducers (which are now effectively reproducers). At that stage almost no survivors are procreated because the maximal level of heredity on the ability to procreate is nearly 1 and the fidelity with which this level is transmitted is also nearly 1 (as shown on figure 5b). If this level is not reached in both cases it is only due to the fact that some minimal reproducers mutate every unit of time (on average 5 individuals per unit of time in a population of 5000) and half of those mutations reduce the average fidelity and average maximal level of transmission of the ability to procreate. 


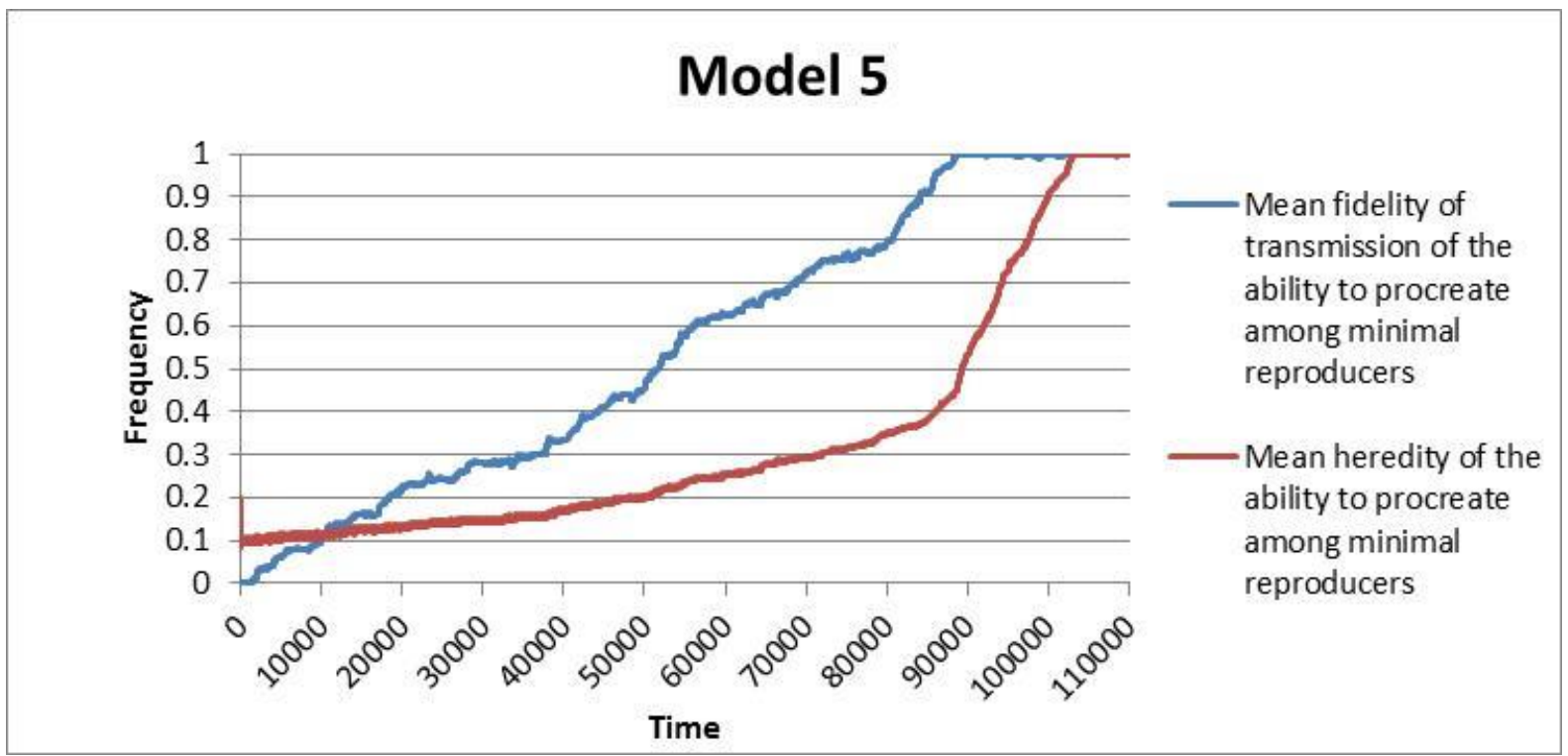

Figure 5b. Simulation of Model 5 over 110000 units of time

Model 5 shows that high heredity of the ability to procreate, as well as high fidelity on the level of transmission of the ability to procreate are both attractors of minimal Darwinian populations. In fact, a population composed mostly of survivors and minimal reproducers with a very low level of heredity of the ability to procreate and very low fidelity of transmission of this ability is invaded by mutants with very high level of heredity and fidelity on this trait and the population becomes a population of reproducers. More interestingly, mutants that overall decrease the number of minimal reproducers produced each unit of time, which represent $50 \%$ of all mutations, seem to play no or only a limited role in the evolutionary dynamics of the population. There is here an asymmetry between mutations increasing or mutations decreasing the level of heredity of and on the ability to procreate. This asymmetry is what makes changes of the population-level background against which new mutations can occur possible.

The explanation underlying the asymmetry is the following. Each time a new mutation increases either the maximal level of heredity of the ability to procreate or the fidelity with which this maximal level is transmitted (i.e. heredity on this ability), this increases the rate of production of minimal reproducers because the minimal reproducers procreated are themselves able to 
To appear in Biology and Philosophy

This version my slightly differ from the final publication

procreate new minimal reproducers. If, on the contrary, a mutation increases the level of production of survivors (via a mutation decreasing heredity of or on the ability to procreate), this mutation will have long-term downstream consequences that will lead the bearers of this mutation to have overall less offspring than the rest of the population and their type will be less represented (and ultimately eliminated) from the population. This, I claim, represents a genuine case of cumulative ENS. Reproduction can thus be a complex (or multiple-step) adaptation in minimal Darwinian populations which emerges as a result of cumulative ENS. Model 5 satisfies requirement $d$, but as such not requirement $c$ because heredity on traits other than the ability to procreate is nil.

In a population in which every individual minimally reproduces, paradigmatic ENS is now extremely easy to get. The only requirement is heredity on traits having consequences for viability or fertility as in Model 4. When this occurs, reproductive cumulative ENS - i.e. paradigmatic ENS - becomes possible and structures like the human eye become in principle much more likely to appear because the number of trials made by natural selection is maximal. To show this, I created Model 6 (see Table 1), which starts with the setup obtained at the end of the simulation of Model 5: a population of reproducers.

In a population of reproducers, as I defined them, the only trait reliably transmitted is the ability to procreate. All other traits are uncorrelated between parents and offspring. Let us now imagine a population of reproducers in which each reproducer is able to mutate, as in Model 5, but on two different traits, one increasing or decreasing its viability, ${ }^{8}$ the other increasing or decreasing the fidelity with which its viability will be transmitted to its offspring. If paradigmatic ENS is possible in such population, we should expect that ultimately the population will be invaded by individuals with the highest possible viability allowed by the model.

${ }^{8}$ A similar mutation could appear and affect fertility. 
To appear in Biology and Philosophy

This version my slightly differ from the final publication

In Model 6, I start with a population of reproducers with viabilities and fertilities all chosen randomly between 0 and 0.99 and 0 and 10 respectively. As in Model 5 there is initially no heredity on viability and fertility. However, unlike Model 5 , individuals can mutate at a $10^{-3}$ rate and increase or decrease their viability by 0.01 (unless the viability is already maximal), but also increase or decrease the fidelity with which viability is transmitted to their offspring, if any, and transmit this level of fidelity to their offspring with the same rate. As in Model 5 the initial population is composed of 5000 individuals. For the sake of simplicity, viability is transmitted as if it were only dependent on one trait (e.g. size), but more complex simulations could be used with viability depending on more traits.

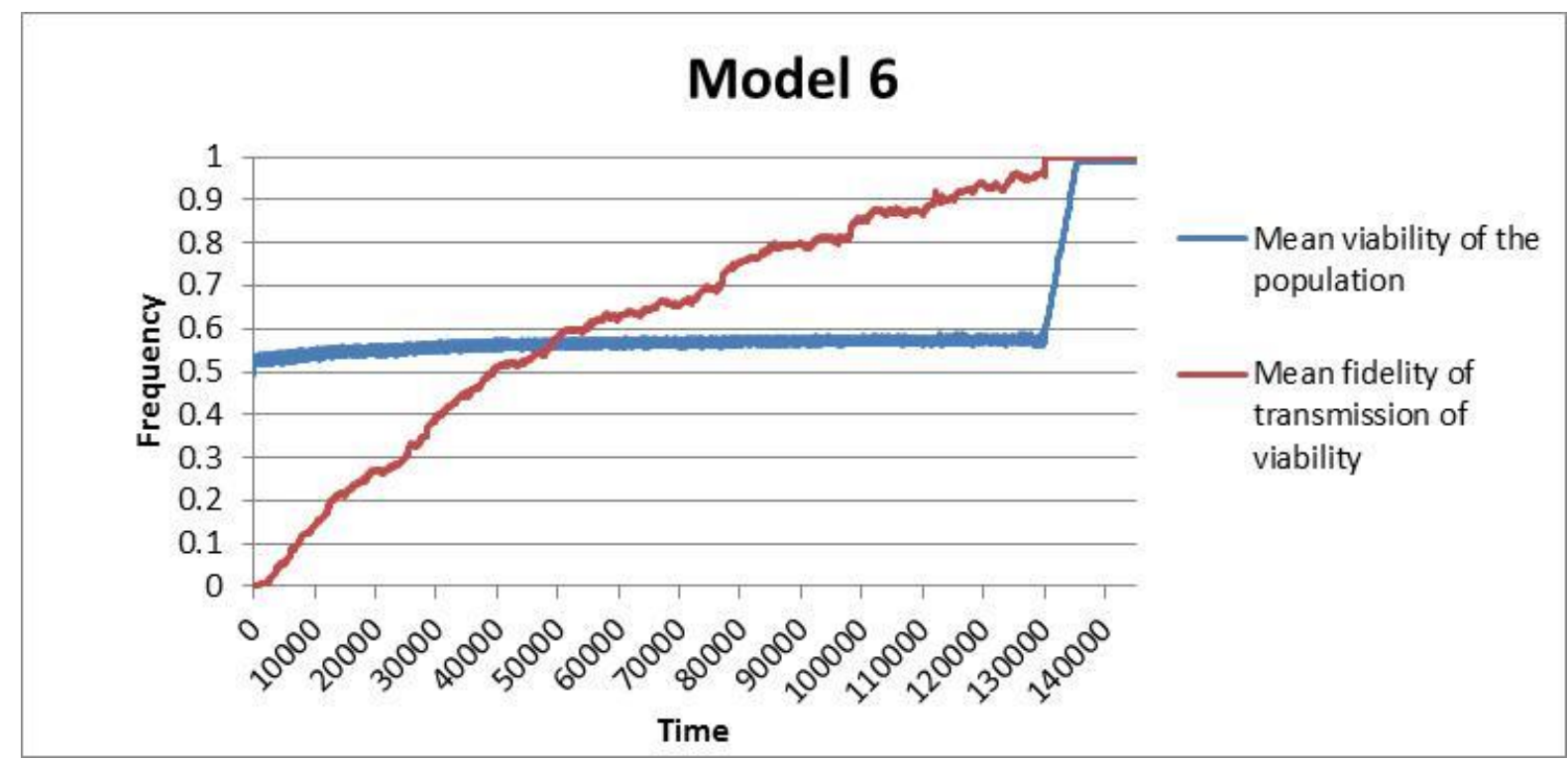

Figure 6. Simulation of Model 6 over 145000 units of time

Model 6 shows two things. First, high heredity on viability can be a complex adaptation of populations of reproducers analogous to reproduction as an adaptation of populations of minimal reproducers. As can be seen in figure 6, although half of the mutations decrease the fidelity of transmission of viability (i.e. heredity on viability) from parent to offspring, after $\mathrm{t}=133000$, fidelity is maximal. This is explained by the fact that reproducers with higher viability 
To appear in Biology and Philosophy

This version my slightly differ from the final publication

have more offspring than reproducers with lower viability when they are able to transmit their viability with a probability higher than chance. This also explains why the mean viability of the population initially slowly increases (from 0.5 to 0.575 at $\mathrm{t}=130000$ in this simulation). As heredity on viability reaches its maximal value, the dynamic of the population radically changes: The mean viability of the population rapidly increases until it reaches the maximal value allowed by the model and remains at that level. The explanation here is exactly the same as previously. The only difference is that reproducers are now replicators. Replicators are able to transmit Darwinian traits to all their offspring with very high fidelity. When that happens, any difference increasing fitness leads to large differences in the population-level background against which new mutations can occur. Indeed, at that point any mutation increasing the viability of replicators is perfectly recorded by natural selection. Increases in viability are also recorded before the appearance of replicators, but the recording is imperfect and thus changes in population level background do not have the dramatic effects they have in populations of replicators.

A further distinction can be made between minimal replicators, replicators and ultimate replicators. Minimal replicators only transmit faithfully one of their traits (aside from the ability to procreate) to their offspring. ${ }^{9}$ Replicators transmit faithfully more than one trait to their offspring, while ultimate replicators are replicators in a classical sense (Dawkins 1982, Hull 1980) and transmit all their traits to their offspring. Further models could show that the selection of a global mechanism of transmission of many, if not all traits, is more advantageous than a successive selection of mechanisms of transmission for each Darwinian trait. Thus structures like DNA with 'unlimited' heredity (Maynard Smith \& Szathmáry 1995, 43) and proofreading during transmission, are expected products of natural selection. I leave this demonstration open to further work.

\footnotetext{
${ }^{9}$ This definition of replicators leads to a form of replication similar to the one argued for by Nanay (2011) who claims that replication is about properties rather than entities.
} 
To appear in Biology and Philosophy

This version my slightly differ from the final publication

Using different models I have shown that reproduction and heredity, usually conceived of as requirements for ENS, are actually predictable products of it, given that new variation is introduced in a population of survivors. By doing so, I have endogeneized reproduction and heredity in a broader conception of ENS.

\section{Assessing the endogenization-of-heredity hypothesis}

The six models I have proposed demonstrate that under very flexible assumptions both reproduction and heredity are likely to appear and spread by natural selection in populations of entities in which those properties are not initially present. It follows that they embody a conception of ENS more abstract than standard conceptions in which reproduction and heredity are taken as necessary conditions for selection to occur. Griesemer (2005) argues that abstractions of Darwinian evolutionary theory take two forms. One consists in abstracting the level at which evolution by natural selection can occur. This is the strategy adopted by Lewontin (1970) in his classical paper "The Units of Selection". The other strategy consists in abstracting away from the contingent mechanisms of biological entities, keeping only the functions that are necessary and sufficient for ENS. Where do the endogenization-of-heredity hypothesis resulting from the 6 models I have proposed stand with regards to Griesmer's two forms of abstraction? In none of the 6 models did I make any assumptions concerning the level at which the mechanisms of viability, heredity of and heredity occur. Nor did I give a precise mechanism of heredity. Thus, these models represent both an abstraction from any specific level of selection and an abstraction away from specific biological mechanisms. They employ both of Griesemer's strategies of abstraction.

Earlier examples of the second strategy of abstraction have concluded that replication is necessary for ENS (Dawkins 1982, Hull 1980). My models demonstrate this conclusion is not 
To appear in Biology and Philosophy

This version my slightly differ from the final publication

justified, since Darwinian origin and distribution explanations are both perfectly possible in populations of survivors rather than replicators. In fact, my endogenization-of-heredity hypothesis represents a plausible Darwinian origin explanation of heredity starting with survivors and assuming some random events of mutation. The endogenization-of-heredity hypothesis shows that the notion of replicator is perfectly dispensable from the notion of ENS.

Some will regard the abstraction instantiated by the six models as justified simply by the fact that it allows the statement of the endogenization-of-heredity hypothesis. Others will wonder what role such a very abstract generalization of Darwinian theory can have in understanding real biological phenomena. In the remainder of this section I explore three areas in which the endogenization-of-heredity model I have proposed could potentially be useful.

The first area in which the endogenization-of-heredity model can be applied is obviously origins of life studies and especially in the study of the origin of evolution by natural selection. Here is not the place to discuss the different theories on the origins of life and the strengths and weaknesses of each of them (for reviews see Cleland forthcoming; Dyson 1999; Fry 2011; Maynard Smith \& Szathmáry 1995). I simply note that the origins of life and the origins of evolution by natural selection, although often treated simultaneously should be treated separately, for a population might be composed of entities considered as alive and yet not undergoing ENS. To a large extent the series of models I have proposed represents a detailed scenario of Dawkins's hypothesis in the second chapter of The Selfish Gene (Dawkins, 1976) where he notes in passing that 'Darwin's 'survival of the fittest' is really a special case of a more general law of survival of the stable" (1976, 13, my emphasis) and that life originated from selection of molecules for stability. But Dawkins jumps very quickly to the idea that through some improbable but possible event a molecule or a complex of molecules must have acquired the ability to self-replicate using small building-blocks available in its surroundings and thus become the first "replicator". The same leap is made by Michod (1999) and by Maynard Smith and 
To appear in Biology and Philosophy

This version my slightly differ from the final publication

Szathmáry (1995) among others. These authors presuppose that evolution becomes an interesting phenomenon (and that life emerges) only when an entity is able to self-replicate with high fidelity. One virtue of the endogenization-of-heredity model I have proposed, is that it shows that ENS can occur and lead to a complex adaptation such as high-fidelity heredity even without high-fidelity replication initially. The replicator-first view of the origins of ENS is thus unwarranted. Whether the scenario I have proposed for the origins of reproduction and heredity is the correct one is not of first importance since my goal was primarily to clarify the space of possibilities with respect to reproduction and heredity. And in fact, procreation, 'heredity of and 'heredity on' might have originated simultaneously, rather than sequentially. But the value of recognizing that there are two different concepts behind what is termed usually referred as "heredity" is demonstrated by the fact that it allows us to see that there are alternative scenarios for this key step in evolution.

Another domain for which the endogenization-of-heredity hypothesis might be applicable is in the broader study of major transition. In the models proposed above, the agents could represent any entities at any level. Procreators and minimal reproducers could represent early multicellular organisms for example. Each primordial multicellular organism would initially fission and would pass on its traits at the collective level more or less randomly from one generation to the other. Collectives would even at that stage be selected for and evolve by natural selection, because the collectives that passed on their traits with more certainty to their offspring would have an advantage and spread.

Finally, the endogeneization-of-heredity hypothesis might be useful in other domains in which the Darwinian apparatus has been deployed, most notably with cultural evolution (GodfreySmith 2009; Mesoudi, Whiten, \& Laland 2004; Richerson \& Boyd 2005). If a replicator is not necessary for ENS in the biological realm, then efforts to find a cultural analog may be unnecessary. This conclusion should be welcomed by cultural evolutionists, since forms of 
To appear in Biology and Philosophy

This version my slightly differ from the final publication

'memetics' relying strongly on the notion of replicator (Blackmore 2000; Dawkins 1976; Dennett 1995) have failed to produce many significant results and their theoretical foundations have been heavily criticized (e.g. Sperber 1996, 2000).

The mere fact that the endogenzization-of-heredity hypothesis raises questions about what counts as Darwinian evolution is important and might lead to further development of the idea.

\section{Conclusion}

In this paper I have shown four things about ENS. First, strictly speaking reproduction is not needed for ENS to occur. Viability selection is sufficient to produce evolutionary changes through the action of natural selection. If reproduction is not necessary, the same can be said about heredity, since the former is a prerequisite for the latter. Second, I have shown that paradigmatic ENS can arise through successive episodes of adaptation in populations of survivors, provided that suitable mutations can arise in these populations. In doing so, I have endogeneized reproduction and heredity into a broader conception of ENS. Third, I have briefly shown that replicators, and specific replicating structures such as DNA, can arise as adaptations in populations of entities that do not reproduce with high-fidelity. Finally, I have shown potential application of the endogenization-of-heredity hypothesis in three domains of current biological research. One implication of these ideas I have not developed and leave for further work is the links between heredity and the concept of fitness. We have seen that even without differences in viability or/and fertility the evolutionary success of entities with different level of heredity on the ability to procreate could be predicted. This seems problematic if a classical definition of fitness based on viability and fertility is used.

\section{References}


This version my slightly differ from the final publication

Blackmore, S. (2000). The meme machine: Oxford Univeristy Press

Bouchard, F. (2004). Evolution, fitness and the struggle for persistence. Unpublished PhD diss., Duke University.

Bouchard, F. (2008). Causal processes, fitness, and the differential persistence of lineages. Philosophy of Science, 75(5), 560-570.

Bouchard, F. (2011). Darwinism without populations: a more inclusive understanding of the "Survival of the Fittest". Studies in History and Philosopby of Science Part C: Studies in History and Philosopby of Biological and Biomedical Sciences, 42(1), 106-114.

Brandon, R. N. (1990). Adaptation and environment. Princeton: Princeton University Press.

Cleland, C. (forthcoming). Conceptual Challenges for Contemporary Theories of the Origin of Life. Current Organic Chemistry(Special Issue on Prebiotic Chemistry).

Darwin, C. (1859). On the origin of species by means of natural selection. London: J. Murray.

Dawkins, R. (1976). The Selfish Gene. Oxford, UK: Oxford University Press.

Dawkins, R. (1982). The Extended Phenotype: The long reach of the gene: Oxford University Press, USA.

Dennett, D. (1995). Darwin's dangerous idea. New York: Touchstone.

Dyson, F. J. (1999). Origins of life: Cambridge University Press.

Endler, J. A. (1986). Natural selection in the wild. Princeton: Princeton University Press.

Fry, I. (2011). The role of natural selection in the origin of life. Origins of Life and Evolution of Biospheres, 41(1), 3-16.

Godfrey-Smith, P. (2009). Darvinian populations and natural selection: Oxford University Press, USA.

Griesemer, J. R. (2000). The units of evolutionary transition. Selection, 1(1), 67-80.

Griesemer, J. R. (2005). The informational gene and the substantial body: On the generalization of evolutionary theory by abstraction. In M. R. Jones \& N. Cartwright (Eds.), Idealization XII: Correcting the Model -Idealization and Abstraction in the Sciences (pp. 59-115). Amsterdam: Rodopi.

Hull, D. (1980). Individuality and selection. Annual Review of Ecology and Systematics, 11(1), 311-332.

Lewontin, R. C. (1970). The units of selection. Annual Review of Ecology and Systematics, 1(1), 1-18.

Lewontin, R. C. (1978). Adaptation. Scientific American, (293), 212-228.

Lewontin, R. C. (1985). Adaptation In R. Levins \& R. Lewontin (Eds.), Dialectics and reductionism in ecology (pp. 65-84). Cambridge, MA: Harvard University Press.

Maynard Smith, J., \& Szathmáry, E. (1995). The Major Transitions in Evolution. Oxford: Freeman \& Co.

Mesoudi, A., Whiten, A., \& Laland, K. N. (2004). Perspective: Is human cultural evolution Darwinian? Evidence reviewed from the perspective of The Origin of Species. Evolution, 58(1), 1-11.

Michod, R. E. (1999). Darwinian dynamics. Princeton: Princeton University Press.

Nanay, B. (2011). Replication without replicators. Synthese, 179(3), 455-477.

Okasha, S. (2006). Evolution and the Levels of Selection: Oxford University Press, USA.

Richerson, P. J., \& Boyd, R. (2005). Not by genes alone: How culture transformed buman evolution. Chicago: University of Chicago Press.

Ridley, M. (1996). Evolution (2nd edition). Oxford: Blackwell

Sanford, D. H. (2011). Determinates vs. determinables. Stanford encyclopedia of philosophy.

Sober, E. (2002). The two faces of fitness Thinking about evolution: historical, philosopbical, and political perspectives. Cambridge: Cambridge University Press.

Sperber, D. (1996). Explaining culture: A naturalistic approach: Cambridge Univ Press.

Sperber, D. (2000). An objection to the memetic approach to culture. Darwinizing culture: the status of memetics as a science, $163-173$.

Van Valen, L. M. (1989). Three paradigms of evolution. Evolutionary theory, 9(1), 1-17.

Wilensky, U. (1999). NetLogo. http://ccl.northwestern.edu/netlogo/ (Version 5.0). Evanston, IL: Center for Connected Learning and Computer-Based Modeling, Northwestern University. 